\title{
The Impact of Nanobiotechnology on the Development of New Drug Delivery Systems
}

\author{
O. Kayser ${ }^{1, *}$, A. Lemke ${ }^{1}$ and N. Hernández-Trejo ${ }^{2}$ \\ ${ }^{1}$ University of Groningen, Groningen University Institute of Drug Exploration, The Netherlands and ${ }^{2}$ Freie Universität \\ Berlin, Institut für Pharmazie, Pharmazeutische Technologie, Biotechnologie und Qualitätsmanagement, Kelchstraße \\ 31, 12169 Berlin
}

\begin{abstract}
Nanotechnology, or systems/devices manufactured at the molecular level, is a multidisciplinary scientific field undergoing explosive development. A part of this field is the development of nanoscaled drug delivery devices. Nanoparticles have been developed as an important strategy to deliver conventional drugs, recombinant proteins, vaccines and more recently nucleotides. Nanoparticles and other colloidal drug delivery systems modify the kinetics, body distribution and drug release of an associated drug. Other effects are tissue or cell specific targeting of drugs and the reduction of unwanted side effects by a controlled release. Therefore nanoparticles in the pharmaceutical biotechnology sector improve the therapeutic index and provide solutions for future delivery problems for new classes of so called biotech drugs including recombinant proteins and oligonucleotides. This review discusses nanoparticular drug carrier systems with the exception of liposomes used today, and what the potential and limitations of nanoparticles in the field of pharmaceutical biotechnology are.
\end{abstract}

\section{INTRODUCTION}

A key area in drug delivery is the accurately targeting of the drug to cells or tissue of choice. Drug targeting systems should be able to control the fate of a drug entering the body. Today's delivery technologies are far away from the design of the so called "magic bullet", proposed by Paul Ehrlich at the beginning of the $20^{\text {th }}$ century, in which the drug is precisely targeted to the exact side of action. Nanotechnology offers here another challenge to come to this goal a bit closer, to deliver the drug in the right place at the right time. But the question is what makes nanotechnology so interesting, and what are present formulation strategies for drugs? A well known strategy is the development of nanoparticles for biomedical and biotechnological applications. Nanoparticles are defined as particles sized below $1 \mu \mathrm{m}$ and can consist out of different biodegradable materials like natural or synthetic polymers, lipids or phospholipids, even metals. The drug can either be integrated in the matrix or attached to the particle surface. Submicron particles possess a very high surface to volume ratios. As a consequence the dissolution rate is increased according to Noyes Whitney and Kelvin equations. For example, poorly soluble compounds like paclitaxel, cyclosporine or amphotericin B show an increased dissolution rate and absorption in the gastrointestinal tract when formulated as nanosuspensions [1]. Depending on the particle charge, surface properties, and relative hydrophobicity, nanoparticles can be designed to adsorb preferentially on organs or tissues. The effectiveness of these nanoparticles has been demonstrated for mucoadhesive systems by Brannon-Peppas [2] for the

\footnotetext{
*Address correspondence to this author at the University of Groningen, Groningen University Institute of Drug Exploration, Antonius Deusinglaan 1, 9713 AV Groningen, The Netherlands; Tel: +31-50-363-3299; Fax: +3150-363-3000; Email: o.kayser@farm.rug.nl
}

gastrointestinal tract and for the blood brain barrier by Gessner et al. [3, 4].

Besides of biopharmaceutical aspects, that are discussed later, the art of encapsulating of drug in so called nanospheres provides protection against agents, which are susceptible to degradation and prolongs exposure of the drug by controlled release. Main disadvantages of nanoscaled particles are difficult production, storage and administration because of physical instability phenomena such as aggregation. On the other hand, the main advantage is their ability to cross membrane barriers, particularly in the CNS and the gastrointestinal tract. Overcoming these barriers gives a deeper understanding of normal and unphysiological processes. Today nanoparticles are developed for further applications such as enzyme immobilization and DNA transfection. In the age of genetic manipulation and somatic gene therapy, transfection systems using nanoscaled particles are custom tailored by the use of designed polymers for specific applications.

This article discusses main approaches in the field of nanotechnology and drug delivery. It should be noted that the scope of this contribution does not allow a detailed discussion of the whole emerging field why we excluded liposomes. But it rather touches mainstream lines to give a picture of important key areas in this fast growing segment of nanotechnology.

\section{NANOSUSPENSIONS AND NANOCRYSTALS}

Nanosuspensions consist of the pure poorly water soluble drug, suspended in an appropriate dispersion media. Production of nanocrystals and related nanosuspensions is called "nanonisation" [5]. The drug powder is homogenised by high pressure homogenisation (piston-gap-technique) [6], wet milling [7] or alternative techniques like nanocrystallisation [8] from supersaturated solution state or spray 
drying [8]. The solid state of resulting nanoparticles is characterised by a high weight per volume, which is an ideal situation for the sustained release as a depot. Drug compounds are best used for the nanosuspension technology, if they form crystals with high energy content, which renders them insoluble in either organic (lipophilic) or hydrophilic media. Nanosuspensions can be used for increasing bioavailability after oral application, e.g. amphotericin B, danazol or tacrolimus. In addition, several solubility related problems of poorly soluble drugs can be resolved, such as a reduced variability of absorption, a faster onset of action, and improved dose proportionality. When given parenteraly as injectable, blood level peaks heights are minimized because of their slowly dissolving process. The drug will be uptaken by the mononuclear phagocytic system to allow regional specific delivery. This can be used for targeting antimycobacterial, fungal, or leishmanicidal drugs to macrophages, if the infectious pathogen is persisting intracellularly. Especially for leishmanicidal drugs like amphotericin B or buparvaquone, this leads to safety improvement, reducing side effects on other organs, this also permits higher dosing and improved efficacy. Products on the market are Rapamune $^{\circledR}$ (Wyeth) and Emend ${ }^{\circledR}$ (Merck) based on the pearlmilling technology licensed by Elan Nanosystems.

\section{SOLID LIPID NANOPARTICLES}

Solid lipid nanoparticles (SLN) were developed at the beginning of the 1990s as an alternative carrier system to emulsions, liposomes and polymeric nanoparticles as a colloidal carrier system for controlled drug delivery. Main reason for their development is the combination of advantages from different carriers systems like liposomes and polmeric nanoparticles. SLN have been developed and investigated for parenteral [9, 10], pulmonal [11] and dermal $[12,13]$ application routes. With the product Nanobase ${ }^{\circledR}$ (Yamanouchi), the first commercial SLN formulation has reached the market recently. Solid Lipid Nanoparticles consist of a solid lipid matrix, where the drug is normally incorporated, with an average diameter below $1 \mu \mathrm{m}$ [10]. To avoid aggregation and to stabilize the dispersion, different surfactants are used that have an accepted GRAS (Generally Recognized as Safe) status. Nanoparticles are also produced by high pressure homogenisation as described for nanosuspensions [14].

SLN have been considered as new transfection agents using cationic lipids for the matrix lipid composition [15, 16]. Cationic solid lipid nanoparticles (SLN) for gene transfer can be formulated using the same cationic lipids as for liposomal transfection agents. In comparison to DOTAPliposomes tested cationic nanoparticles liposomes showed the same transfection rate and gene expression as liposomes. Cationic lipid composition seems to be more dominant for in vitro transfection performance than the kind of colloidal structure it is arranged in. Hence, cationic SLN extend the range of highly potent non-viral transfection agents by one with favourable and distinct technological properties [16].

\section{NANOTUBES AND NANOWIRES}

Nanotubes can be considered as spherical self assembling lipid nanotubes that resemble tiny drinking straws [17]. Main advantage of these spherical nanoparticles is the increased internal volume, and the option to functionalise the inner or external surface. Because of detailed discussion in this issue, only main advantages should be highlighted. The cylindrical geometry allows encapsulation of the drug followed by the fabrication and modification of the vehicle. Drugs can be covalently bound on the surface as demonstrated for testosterone by Goldstein et al. 2001 [18], but mostly remain diffusion controlled in the internal volume. From its dimension nanotubes show a diameter up to $100 \mathrm{~nm}$ and a length from several up to hundreds of microns.

\section{POLYMERIC NANOPARTICLES}

In comparison to SLN or nanosuspensions polymeric nanoparticles (PNPs) consists of a biodegradable polymer. Biocompatibility is an essential feature for potential application as tissue engineering, drug and gene delivery and new vaccination strategies. Most biodegradable polymers consists of synthetic polyesters like polycyanoacrylate [19] or poly(D, L-lactide) and related polymers like poly(lactid acid) PLA or poly(lactide-co-glycolide) to give a few examples. Latest developments also include natural polymers like chitosan [20], gelatine [21, 22], and sodium alginate [23] to overcome some toxicological problems with the synthetic polymers.

Polymeric nanoparticles represent a significant improvement over traditional oral and intravenous methods of administration in terms of efficiency and effectiveness [24]. The advantages of using PNPs in drug delivery are many, being the most important that they generally increase the stability of any volatile pharmaceutical agents and that they are easily and cheaply fabricated in large quantities by a multitude of methods. Also, polymeric nanoparticles may have engineered specificity, allowing them to deliver a higher concentration of pharmaceutical agent to a desired location. Mostly under the term of nanoparticle, nanospheres are understood. From its definition nanospheres are considered as a matrix system in which the matrix in uniformly dispersed. It should be mentioned, that besides of these spheric vesicular systems nanocapsules are also known, where a polymeric membrane surrounds the drug in a matrix core.

The choice of polymer and the ability to modify drug release from polymeric nanoparticles have made them ideal candidates for cancer therapy, delivery of vaccines, contraceptives and delivery of targeted antibiotics. Moreover, polymeric nanoparticles can be easily incorporated into other activities related to drug delivery, such as tissue engineering, and into drug delivery for species other than humans. From the polymer chemistry viewpoint, there will be in the future a challenging field to create new polymers matching hydrophilic and lipophilic properties of upcoming drugs for smart formulation.

\section{NEXT GENERATION OF DRUG DELIVERY SYSTEMS}

The development of nanoparticular systems will be influenced by so called "intelligent material design". Delivery systems will respond the concentration of the drug itself or of certain molecules in the body. These sensitive 
material consists of biomimetic polymers forming a three dimensional network as matrix for the integrated drug. These structures must be flexible enough to allow diffusion of a solvent, biological medium in and out of the polymeric network. Based on the work of micropatterned devices and systems for biological recognition, the versatility and main principle has been demonstrated by Langer and Peppas [25]. But again, nanoparticulate carriers will be integrated with recognitive molecular systems with biosensing properties to detect amino acids, drugs, steroids or DNA via imprinting methods [26]. In the near future biomimetic methods seems to be realistic as indicated by Dillo and Lowmann [27]. An example is the synthesis of biomimetic gels and molecular imprinted release [28]. Using smart polymers for hydrogels that are connected with a biosensor, an autofeedback drug delivery system can be employed to respond to changes from the surrounding environment. The field of microelectronics will also contribute to the expanding field of nanobiotechnology. Electronic devices have been miniaturised and reached a stage of dimension being close biological macromolecules. Even molecular electronics use the same self assembling processes as biomolecules, combining both technologies will lead to the design of ultrafast, ultrasmall and biocompatible devices.

Besides of these new and fascinating properties of nanosized materials, toxicological problems have to be faced and solved in the future. Single dose or short time application over one or two weeks probably will not cause serious health problems. The problems will arise, if poorly soluble particles and non biodegradable particles are used for long term or life time therapy like diabetes, asthma or rheumatoides. Recent work showed for inorganic nanoparticles (polytetrafluoroethylene, $\mathrm{TiO}_{2}$ ) the transport of nasal applied particles through the olfactory mucosa and reached the cerebellum [29]. Main reasons for potential toxicity are explained by their unique size and physical characteristics. Nanoparticles can become toxic because they take on catalytic properties and become biologically engulfable. In contrast to microparticles, cellular uptake of nanoparticles is favoured and standard histological assays showed that pulmonary applied dose of carbon nanoparticles remained intact even after 90 days [30]. If nanoparticulate drug delivery systems become widespread, concerns about nanoparticles' toxicity must be addressed.

The potential nanotechnology in the field pf pharmaceutical biotechnology will positively affect medical and pharmaceutical science in all areas. Improved diagnostics will allow not only working with Point-of-Care devices close to the patients, but also to combine diagnostic and therapeutic actions in a nanoscaled drug delivery systems. Interest and investments in this area will continue to provide contemporary and profound medical applications.

\section{REFERENCES}

[1] Jia, L.; Wong, H.; Cerna, C.; and Weitman, D. (2002) Pharm. Res., 19, 1091-1096.

[2] Brannon-Peppas, L. and Blanchette, J.O. (2004) Adv. Drug. Deliv. Rev., 56, 1649-1659.

[3] Gessner, A.; Olbrich, C.; Schröder, W.; Kayser, O. And Müller, R.H. (2001) Int. J. Pharm., 214, 87-91.

[4] Kreuter, J. (2001) Adv. Drug Deliv. Rev., 47, 65-81.

[5] Rabinow, B.E. (2004) Nat. Rev. Drug Discov., 3, 785-796.

[6] Müller, R.H.; Jacobs, C. and Kayser, O. (2001) Adv. Drug Deliv. Rev., 47, 3-19.

[7] Merisko-Liversidge, E.; Liversidge, G.G. and Cooper, E.R. (2003) Eur. J. Pharm. Sci., 18, 113-120.

[8] Sarkari, M; Brown, J.; Chen, X.; Swinnea, S. and Williams, R.O. $3^{\text {rd }}$ and Johnston, K.P. (2002) Int. J. Pharm., 243, 17-31.

[9] Kipp, J. (2004) Int. J. Pharm., 284, 109-122.

[10] Wissing, S.A.; Kayser, O. and Müller, R.H. (2004) Adv. Drug. Deliv. Rev., 56, 1257-1272.

[11] Fundaro, A.; Cavalli, R.; Bargoni, A.; Vighetto, D.; Zara, G.P. and Gasco, M.R. (2000) Pharmacol. Res., 42, 337-343.

[12] Maia, C.S; Mehnert, W. and Schäfer-Korting, M. (2000) Int. J. Pharm., 196, 165-167.

[13] Dingler, A.; Blum, R.P.; Niehus, H.; Müller, R.H. and Gohla, S. (1999) J. Microencapsul., 16, 751-767.

[14] Schwarz, C. and Mehnert, W. (1999) J. Microencapsul., 16, 205213.

[15] Tabatt, K; Sameti, M.; Olbrich, C.; Müller, R.H. and Lehr, C.M. (2004) Eur. J. Pharm. Biopharm., 57, 155-162.

[16] Tabatt, K; Kneuer, C.; Sameti, M.; Olbrich, C.; Müller, R.H.; Lehr, C.M. and Bakowsky, U. (2004) J. Control. Release, 97, 321-332.

[17] Martin, C.R. and Kohli, P. (2004) Nat. Rev. Drug Discov., 2, 29-37.

[18] Goldstein, A.S.; Amory, J.K.; Martin, S.M.; Vernon, C.; Matsumoto, A, and Yager, P. (2001) Bioorg. Med. Chem., 9, 28192825.

[19] Fattal, E.; Vauthier, C.; Aynie, I.; Nakada, Y.; Lambert, G.; Malvy, C. and Couvreur P. (1998) J. Control. Release, 53, 137-143.

[20] Fernandez-Urrusuno, R.; Calvo, P.; Remunan-Lopez, C.; Vila-Jato, J.L. and Alonso, M.J. (1999) Pharm. Res., 16, 1576-1581.

[21] Farrugia, C.A. and Groves, M.J. (1999) Anticancer Res., 19, 1027 1031.

[22] Farrugia, C.A. and Groves, M.J. (1999) J. Pharm. Pharmacol., 51, 643-649.

[23] Aynie, I.; Vauthier, C.; Chacun, H.; Fattal, E. and Couvreur, P. (1999) Antisense Nucleic Acid Drug. Dev., 9, 301-312.

[24] Soppimath, K.S.; Aminabhavi, T.M.; Kulkarni, A.R. and Rudzinski, W.E. (2001) J. Control. Release, 70, 1-20.

[25] Langner, R. and Peppas, N. (2003) AIChE J., 49, 2990-3006.

[26] Zachary Hilt, J. and Byrne, M. (2004) Adv. Drug Deliv. Rev., 56, 1599-1620.

[27] Dillo, A. and Lowmann, A. (2002) in Biomimetic materials and design: Biointerfacial strategies, tissue engineering and targeted drug delivery, (Lowmann, A., Ed.), Dekker, New York City, pp. 1896-1924.

[28] Byrne, M.E.; Park, K. and Peppas, N. (2002) Adv. Drug Deliv. Rev., 54, 149-161.

[29] Oberdörster, G.; Finkelstein, J.N.; Johnston, C.; Gelein, R.; Cox, C.; Baggs, R. and Elder, A.C. (2000) Res. Rep. Health Eff. Inst., 574; disc 75-86.

[30] Warheit, D.B.; Laurence, B.R.; Reed, K.L.; Roach, D.H.; Reynolds, G.A. and Webb, T.R. (2004) Toxicol. Sci., 77, 117-125. 
Copyright of Current Pharmaceutical Biotechnology is the property of Bentham Science Publishers Ltd. and its content may not be copied or emailed to multiple sites or posted to a listserv without the copyright holder's express written permission. However, users may print, download, or email articles for individual use. 